\title{
C/N ET CONTROLE DE LA PHYSIOLOGIE DES CULTURES DE PHYTOPLANCTON
}

\author{
Jeanne Moal, Jean-François Samain et Jean-René Le Coz
}

Centre Océanologiqué de Bretagne-B.P. 337-29273 Brest

Cédex, France

\section{ABSTRACT}

- Nutrients can be used for induction of carbohydrate or protein metabolism of three unicellular algae, their chemical variations measured by $\mathrm{C} / \mathrm{N}$ ratio. The $\mathrm{C} / \mathrm{N}$ determination and evolution can be used as a control for growth phase and satured conditions. Cultures of routine production have been tested by such a method. The results are discussed. -

\section{INTRODUCTION}

Si dans des conditions de milieux identiques, les différentes espèces d'algues présentent des compositions chimiques voisines (Réf. 1), (Réf. 2), de nombreux travaux ont fait apparâttre la sensibilité de ce matériel aux modifications du milieu. En particulier, la composition chimique des algues varie selon les concentrations de sels nutritifs (Réf. 3) ou la phase de croissance (Réf. 4), (Réf. 5). Ces modifications se traduisent surtout au niveau des glucides et des protéines (Rêf. 6), (Rêf. 7). Af in d'obtenir une nourriture vivante de composition choisie pour l'alimentation des Artemia, Copépodes et larves en expérimentation, nous avons testé la capacité d'adaptation de trois espèces d'algues unicellulaires in vitro: Tetraselmis suecica, Phaeodactylum tricornutum, Monochrysis lutheri. La situation du phytoplancton dans la chaîne alimentaire lui confère une place importante dans les mécanismes des tranferts énergétiques. Si pour la production secondaire, l'importance de la concentration du phytoplancton, de sa composition spécifique, de sa taille est connue, l'incidence de sa qualité chimique reste peu analysée. Pourtant les critères de qualité des algues vivantes sont primordiaux pour la réussite des élevages utilisant celles-ci comme source de nourriture : qualité bactériologique (Réf. 8) et valeur nutritive.

\section{MATERIEL ET METHODES}

La maintenance des cultures est assurée dans des ballons en pyrex de 201 préalablement stérilisés à l'autoclave. Le milieu de culture standard (Réf. 9, tableau 2) est stérilisé par filtration sur filtre Millipore de 0,2 $\mu$. La salle d'élevage est thermostatée a $20^{\circ}$, sous éclairage continu, et l'aération des cultures est assurée par bullage d'air comprimé enrichi en $\mathrm{CO}_{2}$ (Réf. 10). Un quart de la culture est quotidiennement renouvelé, en substituânt stérilement 51 de culture par 51 de solution nutritive.

Plusieurs paramètres permettant de suivre l'évolution de la culture, ont été analysés sur des prélèvements journaliers. Une numération cellulaire est effectuée au microscope inversé Reichert en utilisant une cellule de Malassez. 
La composition en carbone et azote a été mesurée sur l'analyseur C.H.N. Hewlett Packard. Un volume connu de la culture est recueilli sur filtre en fibre de verre Whatman GF/C de $25 \mathrm{~mm}$. Les nitrates et les phosphates ont été déterminés à 1 'autoanalyseur Technicon, sur le surnageant des cultures après centrifugation, selon la méthode Tréguer et Le Corre (11).

Pour les dosages de protéines, carbohydrates totaux, sucres réducteurs, un volume connu de la culture est centrifugé, le surnageant éliminé et le culot repris dans un volume d'eau distillée, dix fois inférieur à celui de départ. Cette solution est aussitôt broyêe aux ultra-sons pour 1'extraction des composants cellulaires. Le dosage des protéines est effectué sur le surnageant du broyat après centrifugation, selon la méthode de Lowry, adaptée au technicon par Samain et Boucher (12). Sur le broyat total, les carbohydrates totaux ont été mesurés selon la méthode de Dubois (13), adaptée au phytoplancton par Charra Mallara (14), et les sucres réducteurs selon la méthode colorimétrique de Willstätter (15) à l'acide 3,5 dinitrosalícylique. L'amidon est dosé par le réactif iodo-ioduré après trois extractions du broyat dans du tampon phosphate $\mathrm{pH}$ 6,8 (Réf. 16).

\section{RESULTATS}

Expression de la composition chimique. Les travaux de Parsons et al (1) ont montré la possibilité d'exprimer les variations de la composition chimique de diverses algues unicellulaires par l'analyse des valeurs relatives du carbone, de l'azote et du phosphore. Dans un premier temps, nous avons cherché à exprimer les variations des composants cellulaires par le $\mathrm{C} / \mathrm{N}$, dont la détermination peut être envisagée en routine. Les mesures de quelques composés cellulaires tels que carbohydrates totaux, protéines, amidon, acides aminés ont été effectuées parallèlement à des mesures d'azote et de carbone sur des ballons de production de Tetraselmis, Phaeodactylum et Monochrysis. La corrélation entre le C/N et le rapport carbohydrates/protéines est positive et significative au seuil de $5 \%(r=0,98)$. L'existence de cette corrélation permettra $I^{\prime} u t i l i s a t i o n ~ d u ~ C / N$ en tant qu'expression globale des variations relatives des carbohydrates et des protéines.

Orientation du métabolisme de Tetraselmis. Les grandes variations du $\mathrm{C} / \mathrm{N}$, en relation avec le rapport carbohydrates/protéines sont à rapprocher des résultats obtenus par Antia (6), Myklestad (4) qui ont souligné l'importance des quantités et qualités des sels nutritifs présents sur la composition chimique des algues, en particulier sur leur teneur en carbohydrates et en protéines. Dans cette optique, 1 'influence des variations de concentrations des sels nutritifs sur le $\mathrm{C} / \mathrm{N}$ de Tetraselmis a été recherchẻe.

Six cultures continues de Tetraselmis ont étê effectuées en présence de concentrations de sels nutritifs de 0,$5 ; 1 ; 2,5 ; 5 ; 10$ et 50 fois les teneurs de la solution standard. La concentration en vitamines n'a pas été modifiée. $L$ 'évolution du $\mathrm{C} / \mathrm{N}$ pendant 10 jours est présentée dans $1 \mathrm{a}$ figure (1). Trois niveaux moyens se distinguent couvrant une gamme de $\mathrm{C} / \mathrm{N}$ allant de 5 à 20 . Le niveau inférieur est obtenu dès la concentration 2,5 caractérisant alors un phénomène de saturation ( $f i g$. 2). Contrairement aux observations effectuées sur les cultures. non saturées (milieux 0,5 et 1) les valeurs de $\mathrm{C} / \mathrm{N}$ sont stables et faibles dans les conditions saturées, traduisant une physiologie mieux établie. L'analyse journalière des nitrates et phosphates montre que les milieux 0,5 et 1 sont épuisés en $24 \mathrm{~h}$ et qu'une accumulation progressive des sels se produit dans les milieux 5 et 10 , confirmant le caractère saturant de ces conditions; le milieu 2,5 étant à la limite du phénomène d'accumulation (fig.3) 


\section{$\mathrm{C} / \mathrm{N}$ et controle de la physiologie}
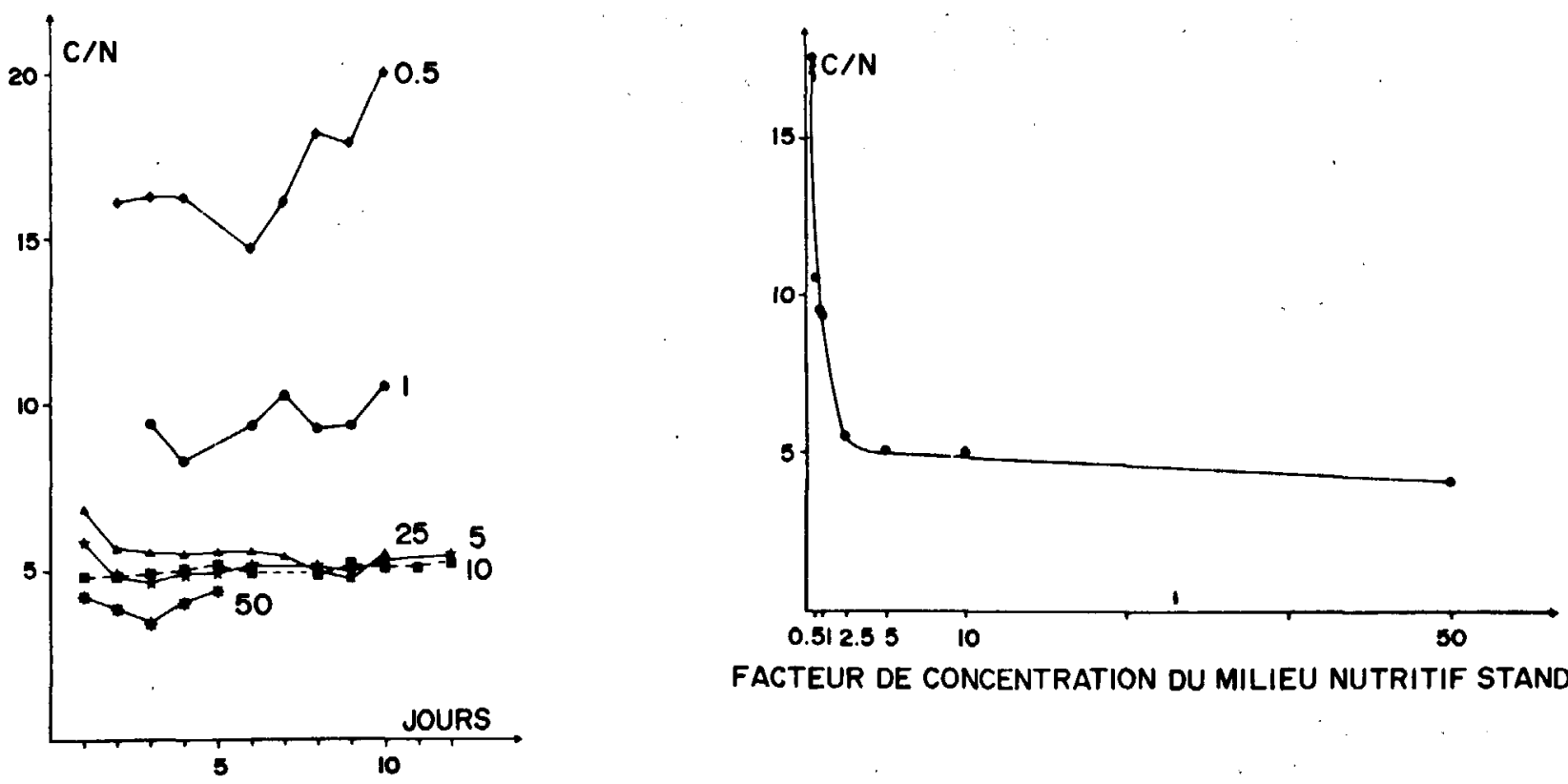

FACTEUR DE CONCENTRATION DU MILIEU NUTRITIF STANDARO

Fig. 1 et 2 : C/N de Tetraselmis en culture continue, aux concentrations 0,5 $1-2,5-5-10-50$ du milieu nutritif standard.

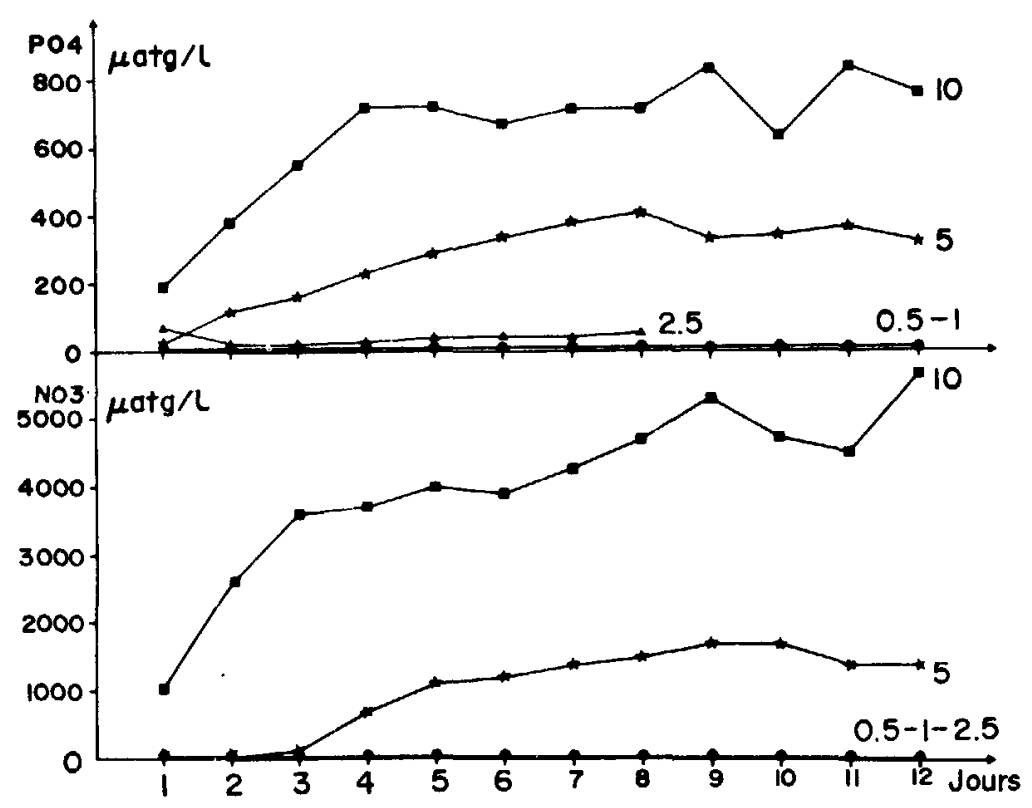

Fig. 3 : Cultures continues de Tetraselmis : concentrations résiduelles des nitrates et des phosphates aux concentrations $0,5-1-2,5-5-10-50$ du milieu nutritif standard.

Par contre, le milieu 50 est devenu rapidement limitant pour 1a croissance ; les cellules se sont rapidement décolorées et enkystées sous 1 'effet probable des concentrations excessives en sels.

La modification progressive du $\mathrm{C} / \mathrm{N}$ à travers les différents niveaux observés peut être suivie sur les cultures 1,5 et 10 dont 1 'enrichissement journalier est supprimé. La concentration cellulaire, les concentrations de nitrates et phosphates ainsi que le rapport $\mathrm{C} / \mathrm{N}$ ont été contrôlés pendant 15 jours. La phase exponentielle de croissance est d'autant plus longue et la concentration cellulaire élevée que la concentration initiale en sels était importante (fig. 4). Le $\mathrm{C} / \mathrm{N}$ augmente dès que la concentration en sels devient inférieure au niveau de saturation et.varie dans la gamme de 5 à 15 . 


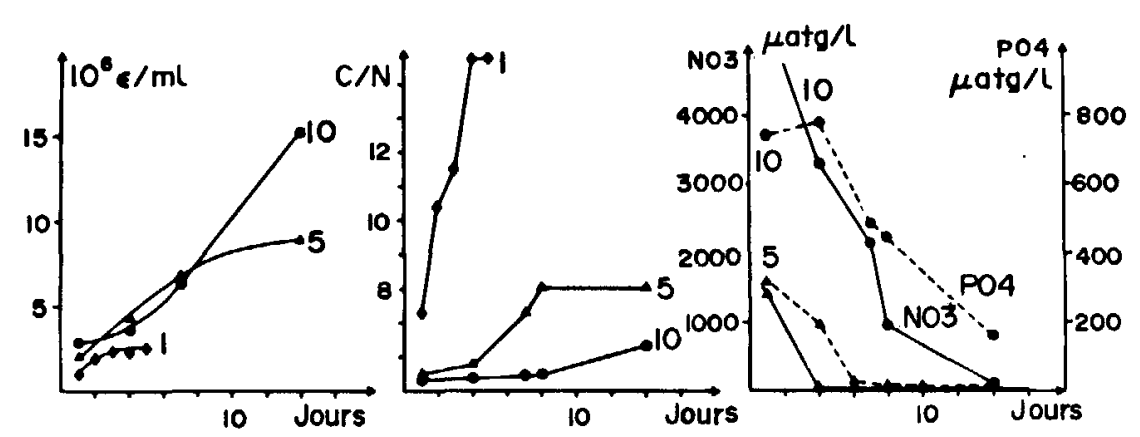

Fig. 4 : Cultures de Tetraselmis en milieu non renouvelé : concentration cellulaire, $\mathrm{C} / \mathrm{N}$, sels nutritifs pour trois concentrations initiales.

La composition chimique des cellules phytoplanctoniques des milieux 0,5 ; 1 et 2,5 a été analysée sur une dizaine de jours. Les résultats exprimés en $\mu \mathrm{g} / 10^{6}$ cellules sont rassemblés dans le tableau 1. La composition chimique des cultures continues 0,5 et 1 est très semblable. La culture 2,5 se distingue par des teneurs faibles en carbohydrates et en amidon, des teneurs supérieures en azote, en protéines et en acides aminés. L'augmentation des carbohydrates dans les milieux 0,5 et 1 est principalement liée à celle de l'amidon dont la proportion passe de 15 à $35 \%$.

\begin{tabular}{|l|c|c|c|c|c|c|c|}
\hline & $\mathrm{C}$ & $\mathrm{N}$ & $\mathrm{C} / \mathrm{N}$ & $\begin{array}{c}\text { Carbohy- } \\
\text { drates }\end{array}$ & Protéines & $\begin{array}{c}\text { Acides } \\
\text { aminés_s }\end{array}$ & Amidon \\
\cline { 2 - 2 } & 82 & 7,4 & 11,1 & 72,8 & 28,1 & 5,7 & 24,1 \\
Milieu 1 & 83,1 & 8,8 & 9,5 & 69,3 & 31,7 & 11 & 24,8 \\
Milieu 2,5 & 99,5 & 18,2 & 5,5 & 22,2 & 54,1 & 14 & 3,4 \\
\hline
\end{tabular}

Tableau 1 : Composition chimique de Tetraselmis ( $\mu \mathrm{g} / 10^{6}$ cellules) aux concentrations 0,$5 ; 1 ; 2,5$ du milieu nutritif standard.

Variabilité comparée du C/N de Tetraselmis, Phaeodactylum et Monochrysis. L'évolution du $\mathrm{C} / \mathrm{N}$ en relation avec l'épuisement en sels a été étudiée sur trois cultures en phase exponentielle : Tetraselmis, Phaeodactylum et Monochrysis, dont les milieux ne sont plus renouvelés. La variabilité intraspécifique du $\mathrm{C} / \mathrm{N}$ est aussi importante chez les trois espèces. Cependant, et bien que les milieux nutritifs soient identiques au départ, les courbes de croissance sont très différentes (fig. 5).

Contrôle des cultures.: Les résultats précédents permettent de mettre en évidence $1^{\top}$ extrême sensibilité de la composition chimique des cellules phytoplanctoniques aux variations de milieu. Le rapport $\mathrm{C} / \mathrm{N}$ fournit une information suffisante pour détecter ces modifications de composition. Il a donc été mesuré systématiquement sur des cultures de routine effectuées au laboratoire de production d'algues du COB. Trois espèces, destinées à l'alimentation des larves de poissons, des Artemia et du zooplancton en expérimentation, y sont cultivées. 

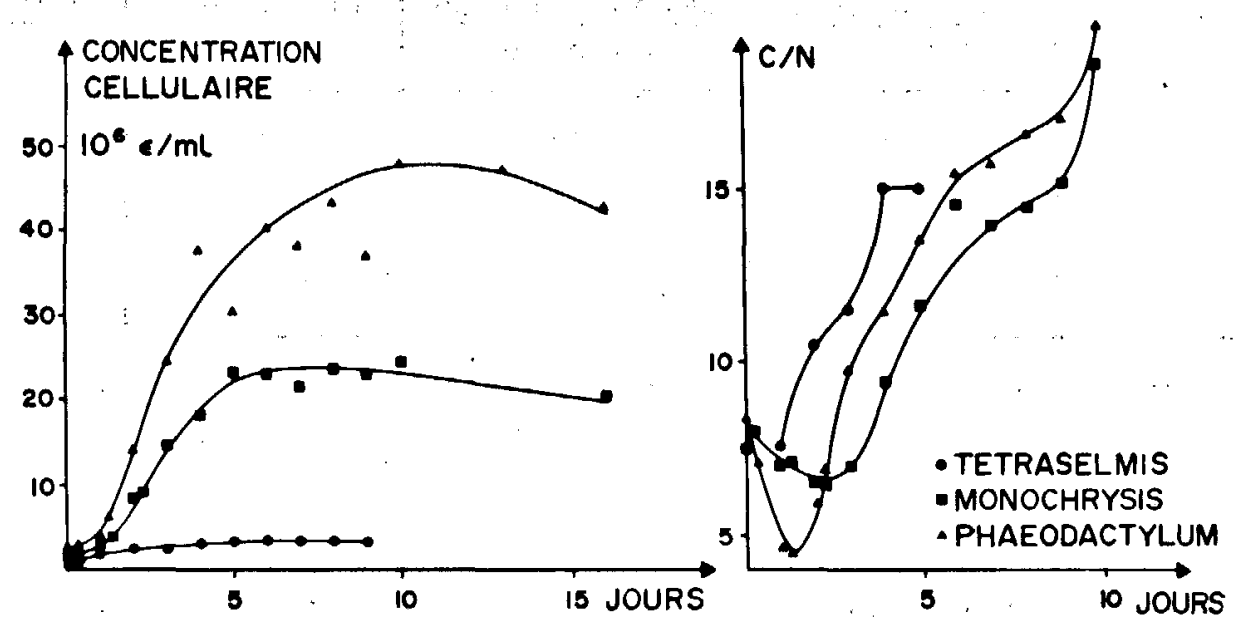

Fig. 5 : Concentrations cellulaires, $\mathrm{C} / \mathrm{N}$ de 3 espèces phytoplanctoniques en milieu nutritif standard non renouvelé.

Les valeurs moyennes du $\mathrm{C} / \mathrm{N}$ et des principaux composés cellulaires, ont été déterminées. La cinétique d'évolution du rapport carbone sur azote en réponse à l'épuisement provoqué du milieu nutritif a été suivie afin de déterminer l'influence d'éventuelles fluctuations du milieu sur la stabilité des cultures. Les valeurs du $\mathrm{C} / \mathrm{N}$ des trois espèces sont soumises à une forte dispersion. Monochrysis présente une valeur de $\mathrm{C} / \mathrm{N}$ moyen $(8,6 \pm 0,6)$ significativement plus basse que les deux autres espèces Tetraselmis $(12,4 \pm 1,1)$, PhaeodactyZum $(11,8 \pm 0,9)$. Sa composition élevée en protéines et faible en carbohydrates confirme cette donnée (tableau 2). Par contre, si Tetraselmis ne se distingue pas de Phaeodactylum par son $\mathrm{C} / \mathrm{N}$, elle a une teneur significativement plus élevée en carbohydrates. L'analyse des glucides ne révèle de 1 'amidon que pour Tetraselmis (43\%). Des sucres réducteurs n'ont été décelés que chez Phaeodactylum (23\%). A 1 'absence de renouvellement du milieu pendant deux jours, on observe une réponse très différente du $\mathrm{C} / \mathrm{N}$ des trois espèces (fig. 6). La variation du $\mathrm{C} / \mathrm{N}$ est très.rapide pour Tetraselmis et Phaeodactylum. Par contre Monochrysis semble peu affectée. L'analyse des concentrations finales en sels pendant cette période montre que seule cette espèce n'a pas épuisé le milieu nutritif.

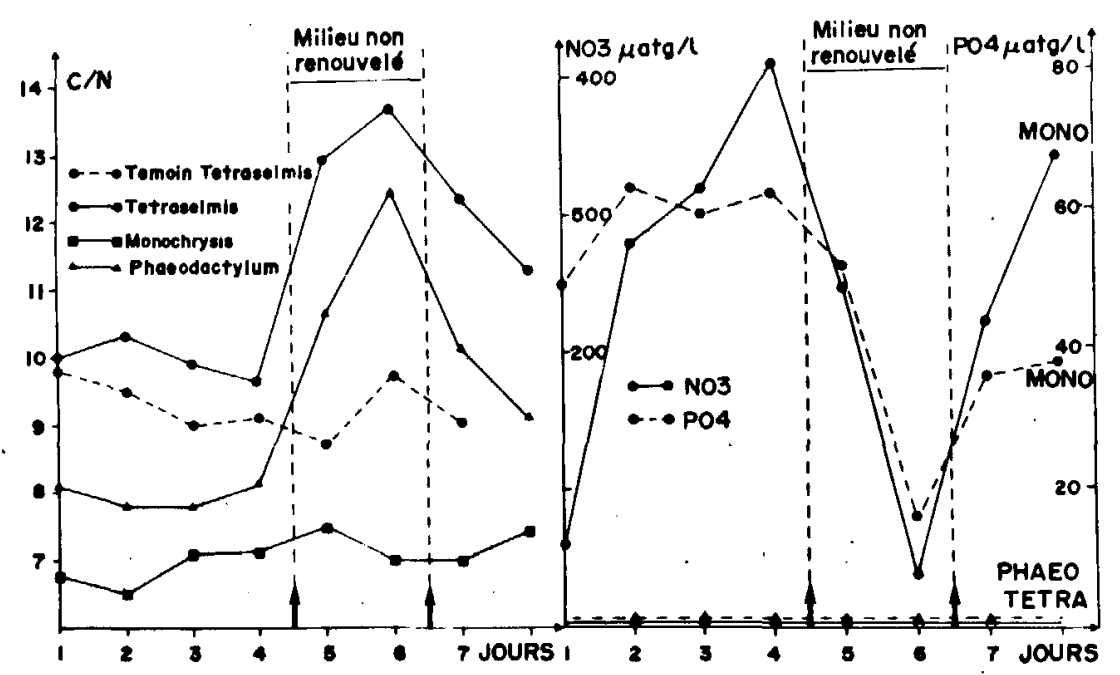

Fig. 6 : Culture continue de 3 espèces : cinétique de réponse du $\mathrm{C} / \mathrm{N}$ et des concentrations de $\mathrm{NO}_{3}$ et $\mathrm{PO}_{4}$ à un non renouvellement du milieu (jours 5 et 6) 


\begin{tabular}{|l|c|c|c|c|}
\hline & $\frac{\text { Protéines }}{\text { Carbone }}$ & $\frac{\text { Carbohydrates }}{\text { Carbone }}$ & $\frac{\text { Protéines }}{\text { Azote }}$ & $\frac{\text { Carbohydrates }}{\text { Azote }}$ \\
\cline { 2 - 5 } & 0,52 & 0,57 & 4,36 & 4,8 \\
Phaeodactylum & 0,36 & 0,87 & 4,27 & 10,3 \\
Tetraselmis & 0,36 & 1,46 & 4,3 & 17,6 \\
\hline
\end{tabular}

Tableau 2 : Composition chimique des trois espèces phytoplanctoniques dans le milieu standard.

\section{$\underline{\text { DISCUSSION }}$}

La grande variabilité du $\mathrm{C} / \mathrm{N}$ en réponse à des modifications du milieu et la corrélation positive entre $1 \mathrm{e} \mathrm{C} / \mathrm{N}$ et le rapport carbohydrates/protéines suggèrent des adaptations du métabolisme. Ces résultats montrent qu'il est possible d'orienter la composition chimique des trois cultures de phytoplancton étudiées en modifiant la concentration en sels nutritifs dans le milieu.

L'enrichissement du milieu en sels nutritifs se traduit par une diminution du rapport $\mathrm{C} / \mathrm{N}$ jusqu'à un seuil minimum $(\approx 5)$ à partir duquel les sels s'accumulent et $1 \mathrm{e} C / \mathrm{N}$ reste stable. L'abaissement du $\mathrm{C} / \mathrm{N}$ correspond surtout à une augmentation d'azote (tableau 1). En effet, bien que les carbohydrates diminuent et les protéines et acides aminés augmentent, le bilan carboné reste identique. Les protéines et acides aminés bien que plus abondants, ont cependant une participation moindre dans 1 'azote total des cellules; 1 'augmentation d'autres composés azotés tels que les acides nucléiques ou les sels minéraux $\left(\mathrm{NH}_{4}-\mathrm{NO}_{3}\right)$ pourrait expliquer ce résultat. L'enrichissement d'acides nucléiques devrait conduire à des multiplications plus rapides. Ceci n'apparaît pas dans les cultures 0,$5 ; 1$ et 2,5 qui ont le même taux de croissance. Il s'agit donc probablement d'une accumulation de sels azotés dans les cellules végétales, ce qui confirme les rêsultats de Conover (7).

L'influence de 1'épuisement des sels se répercute sur les phases de la croissance cellulaire ainsi que sur le $\mathrm{C} / \mathrm{N}$ (fig. 4 et 5 ). Une concentration saturante en sels favorise le maintien en phase exponentielle et une faible valeur $\mathrm{du} \mathrm{C} / \mathrm{N}$; à l'épuisement progressif du milieu correspond l'amorce de la phase stationnaire et 1 'évolution $d u C / N$ vers des valeurs élevées. Le $\mathrm{C} / \mathrm{N}$ peut être un bon indicateur des phases de croissance d'une culture phytoplanctonique et de ce fait permet d'en effectuer le contrôle. Les variations du $\mathrm{C} / \mathrm{N}$ durant les phases de croissance confirment les données signalées par Myklestad (4), Skoglund (17) selon lesquels des cultures jeunes en phase exponentielle possèdent peu de carbohydrates par rapport à des cellules en phase stationnaire. Des études, in vivo, effectuées par Le Masson (18) en Atlantique aboutissent aux mêmes conclusions : des eaux jeunes et riches possèdent un $\mathrm{C} / \mathrm{N}$ plus bas que des eaux anciennes et pauvres.

L'ensemble des résultats permet de confirmer que les variations intraspécifiques du $\mathrm{C} / \mathrm{N}$ sont plus importantes que les variations interspécifiques et qu'il est possible de contrôler l'état physiologique des cultures par la mesure des valeurs moyennes du $\mathrm{C} / \mathrm{N}$ et la vitesse de réponse de cet indice à 1 'absence d'apport de sels nutritifs. Cette vitesse permet de juger de la saturation des conditions du milieu. La richesse des algues en carbohydrates et en 
protéines est orientable par intervention soit sur la composition du milieu en sels nutritifs, soit sur le protocole de renouvellement des milieux de culture. Dans le choix de la culture continue ou discontinue, 1'expérimentateur doit tenir compte de la différence de qualité chimique qui peut en résulter. Le contrôle d'une culture discontinue (fig. 5) permet d'enregistrer des $\mathrm{C} / \mathrm{N}$ pouvant varier sur toute $1 \mathrm{la}$ gamme ( 5 à 20 ). Une telle culture permet d'obtenir à la demande des algues de qualité chimique variée.

La réponse du $\mathrm{C} / \mathrm{N}$ aux modifications de sels nutritifs varie selon les espèces. Pour des conditions de milieux semblables, la composition chimique et le $\mathrm{C} / \mathrm{N}$ peuvent être différents (tableau 2, fig. 6). De plus, la présence d'amidon chez Tetraselmis, même aux fortes teneurs en sels, ou de sucres réducteurs seulement chez Phaeodactylum, paraît correspondre à une spécificitê des algues quant à la synthèse de leurs glucides.

Dans les cultures de routine du laboratoire de production d'algues, la valeur du $\mathrm{C} / \mathrm{N}$ pour les espèces Tetraselmis et Phaeodactylum, varie entre 8 et 13 ; 1 'augmentation de ce rapport est rapide à 1 'absence de renouvellement de milieu. Ces caractéristiques traduisent des conditions limitantes et la possibilité d'un vieillissement rapide de la culture en cas de déséquilibre des sels nutritifs. Cette valeur de $\mathrm{C} / \mathrm{N}$ est supérieure à celle que $I^{\prime}$ on a obtenu pour Tetraselmis dans les mêmes conditions de milieu en culture continue $(C / N=9,5)$ (tableau 1). Ceci est expliqué par le protocole de production de routine au cours duquel aucun renouvellement de milieu n'est effectué pendant le week-end ce qui induit des augmentations périodiques de $\mathrm{C} / \mathrm{N}$. Associée à des conditions limitantes de milieu nutritif, cette technique fournit une production variable en qualité chimique, orientée principalement sur des a1gues en phase préstationnaire, riches en carbohydrates et de faibles teneurs protéiques. La culture de Monochrysis, dans les mêmes conditions, a un $\mathrm{C} / \mathrm{N}$ moyen inférieur $(\simeq 8)$ qui varie lentement à l'absence de renouvellement de milieu. Ce comportement traduit des conditions proches de la saturation en sels, une fin de phase exponentielle confirmée par une teneur protéique plus importante et des carbohydrates en quantité plus faible.

\section{REMERCIEMENTS}

Nous remercions pour leur active collaboration et leurs conseils : J.P. FLASSCH J.C. ALEXANDRE, G. SALAUN, P.Y. GUENOLE, du laboratoire de production d'algues du C.0.B., qui ont pris en charge la réalisation des cultures.

\section{REFERENCES}

(1) PARSONS, T.R., STEPHENS, K., STRICKLAND, J.D.H., 1961. On the chemica1 composition of eleven species of marine phytoplankters. Journal of Fisheries Research Board of Canada, 18, 1001-1016.

(2) RICKETTS, T.R., 1966. On the chemical composition of some unicellular algae. Phytochemistry, 5, 67-76.

(3) HOBSON, L.A., PARISER, R.J., 1971. The effect of inorganic nitrogen on macromolecular synthesis by Thalassiosira fluviatizis Husted and Cyclotella nana Hustedt grown in batch culture. Journal of experimental marine biology and ecology, 6, 71-78. 
J. Moal, J-F. Samain and J-R. Le Coz

(4) MYKLESTAD, S., HAUG, A., 1972. Production of carbohydrates by the marine diatom Chaetoce ros affinis var Wizlei (gran) Hustedt. Journal of experimental marine biology and ecology, 9, 125-136.

(5) BERLAND, B.R., BONIN, D.J., DAUMAS, R.A., LABORDE, P.L., MAESTRINI, S.Y., 1970. Variations du comportement physiologique de 1 'algue Monalzantus salina en culture. Marine Biology, 7, 82-92.

(6) ANTIA, N.J., MC ALLISTER, C.D., PARSONS, T.R., STEPHENS, K., STRICKLAND, J. D.H., 1963. Further measurements of primary production using a large volume plastic sphere. Limnology and Oceanography, 8, 166-183.

(7) CONOVER, S.A.M., 1975. Partitioning of nitrogen and carbon in cultures of the marine diatom Thalassiosira fluviatilis supplied with nitrate, ammonium or urea. Marine Biology, 32, 231-246.

(8) LUCAS, A., PRIEUR, D., 1974. Le contrôle bactérien des élevages de larves de bivalves. Colloque sur $1^{\prime}$ Aquaculture. Actes et Colloques, $n^{\circ} 1,1-24$, CNEXO Ed.

(9) WALNE, P.R., 1966. Experiments in the large scale culture of the larvae of Ostrea edulis (L.). Fishery investigations, London, Ser 2, 25 (4), $1-53$.

(10) FLASSCH, J.P., NORMANT, Y., 1974. Mise en place d'une unité de production d'algues au Centre Océanologique de Bretagne : premiers résultats. Colloque sur $1^{\prime}$ Aquaculture. Actes et Colloques, $n^{\circ} 1,25-19$, CNEXO Ed.

(11) LE CORRE, P., TREGUER, P., 1976. Contribution à l'étude de la matière organique dissoute et des sels nutritifs dans $I^{\prime}$ eau de mer. Thèse doctorat ès-Sciences, Université de Bretagne Occidentale.

(12) SAMAIN, J.F., BOUCHER, J., 1974. Dosage automatique de l'amylase et des protéines du zooplancton. Annales de l'Institut Océanographique, Paris, $50,199-205$.

(13) DUBoIS, M., GILlES, K.A., HAMILTON, J.K., REBERS, P.A., SMITH, F., 1956. Colorimetric method for determination of sugars and related substances. Analytical Chemistry, 28, 350-356.

(14) MALARA, G., CHARRA, R., 1972. Dosage des glucides particulaires du phytoplancton selon la méthode de Dubois. Notes de travail $\mathrm{n}^{\circ} 6$. Université de Paris VI. Station Zoologique de Villefranche-sur-Mer.

(15) WILLSTATTER, R., WALDSCHMIDT-LEITZ, E., HESSE, A.R.F., 1923. Z. Physiologie Chem., 126,143 .

(16) MARTIN, A.G., 1976. Etude de la matière organique particulaire. Thèse 3ème cycle. Université de Bretagne Occidentale.

(17) SKOGLUND, L., JENSEN, A., 1976. Studies of N. limited growth of diatoms in dialysis culture. Journal of experimental marine biology and ecology, $21,169-178$.

(18) LE MASSON, L., CREMOUX, J.L., MONTEL, Y., 1977. Analyse des rapports C/N/ $P$ du seston dans la partie orientale de l'Atlantique équatorial. Marine chemistry, 5, 171-181. 